\title{
MAPPING PROPERTIES OF MULTILINEAR FRACTIONAL MAXIMAL OPERATORS IN METRIC MEASURE SPACES
}

\author{
Feng LiU, Seongtae Jhang, Rui Bu and Zunwei Fu*
}

\begin{abstract}
In this paper, we introduce two kinds of multilinear fractional maximal operators in metric measure spaces. We prove that these operators map product Morrey spaces to Morrey spaces, and map product Lebesgue spaces to the fractional Hajłasz spaces under certain restrictions on the underlying metric measure space. We also introduce a kind of discrete multilinear fractional maximal operator, which is constructed in terms of coverings and partitions of unities and has better regularity. With the aid of Poincare inequality, we establish the Sobolev bounds for the above operators.
\end{abstract}

Mathematics subject classification (2010): Primary 42B25; Secondary 46E35.

Keywords and phrases: Multilinear fractional maximal operator, Morrey space, fractional Hajłasz space, Sobolev space, metric measure space.

\section{REFERENCES}

[1] D. Aalto And J. Kinnunen, The discrete maixmal operator in metric spaces, J. Anal. Math. 111 (2010), 369-390.

[2] B. T. ANh AND X. T. DuOng, On commutators of vector BMO functions and multilinear singular integrals with non-smooth kernels, J. Math. Anal. Appl. 371 (2010), 80-94.

[3] A. BJöRn AND J. BJÖRn, Nonlinear Potential Theory on Metric Spaces, European Mathematical Society, Tracts in Mathematics 17, 2011.

[4] S. M. BUCKLEY, Is the maximal function of a Lipschitz function continuous?, Ann. Acad. Sci. Fenn. Math. 24 (1999), 519-528.

[5] F. Chiarenza and M. Frasca, Morrey spaces and Hardy-Littlewood maximal function, Rend. Mat. Appl. 7 (3-4) (1988), 273-279.

[6] R. R. Coifman And G. Weiss, Analyse Harmonique Non-Comutative sur Certain Espaces Homogènes, Lecture Notes in Mathematics No. 242, Springer-Verlag, 1971.

[7] P. HajŁasz, Sobolev spaces on an arbitrary metric space, Potential Anal. 5 (4) (1996), 403-415.

[8] P. HAJŁASZ, Sobolev spaces on metric-measure spaces, In: Heat kernels and analysis on manifolds, graphs, and metric spaces (Paris, 2002), pp. 173-218, Contemp. Math. 338, Amer. Math. Soc. Providence, RI, 2003.

[9] P. HajŁasz and P. Koskela, Sobolev met Poincaré, Mem. Amer. Math. Soc. 145 (688) (2000).

[10] T. Heik Kinen, J. Kinnunen, J. Nuutinen And H. Tuominen, Mapping properties of the discrete fractional maximal operator in metric measure spaces, Kyoto J. Math. 53 (3) (2013), 693-712.

[11] T. HEIK KINEN AND H. TUOMINEN, Smoothing properties of the discrete fractional maximal operator on Besov and Triebel-Lizorkin spaces, Publ. Mat. 58 (2) (2014), 379-399.

[12] T. Heik KInen, J. Lehrb ÄCK, J. NUUtinen AND H. TUOMInEn, Fractional maximal functions in metric measure spaces, Anal. Geom. Met. Spaces 2013 (2013), 147-162.

[13] S. Keith And X. Zhong, The Poincaré inequality is an open ended condition, Ann. Math. 167 (2008), 575-599.

[14] J. Kinnunen and V. Latvala, Lebesgue points for Sobolev functions on metric spaces, Rev. Mat. Iberoam. 18 (3) (2002), 685-700.

[15] J. Kinnunen And H. Tuominen, Pointwise behavior of $M^{1,1}$ Sobolev functions, Math. Z. 257(3) (2007), 613-630. 
[16] A. K. Lerner, S. Ombrosi, C. Pérez, R. H. Torres and R. Trujillo-González, New maximal functions and multiple weights for the multilinear Calderón-Zygmund theory, Adv. Math. 220 (2009), 1222-1264.

[17] F. LiU AND H. WU, On the regularity of the multisublinear maximal functions, Canad. Math. Bull. 58 (4) (2015), 808-817.

[18] Y. LU, D. YAng AND W. YuAn, Morrey-Sobolev spaces on metric measure spaces, Potential Anal. 41 (2014), 215-243.

[19] Y. Mizuta, T. Shimomura And T. Sobukawa, Sobolev's inequality for Riesz potentials on functions in non-doubling Morrey spaces, Osaka J. Math. 46 (2009), 255-271.

[20] E. Routin, Distribution of points and Hardy type inequalities in spaces of homogeneous type, J. Fourier Anal. Appl. 19 (5) (2012), 877-909.

[21] Y. SAWANO, Sharp estimates of the modified Hardy-Littlewood maximal operator on the nonhomogeneous space via covering lemmas, Hokkaido Math. J. 34 (2) (2005), 435-458.

[22] N. ShanmUgalingam, Newtonian spaces: an extension of Sobolev spaces to metric measure spaces, Rev. Mat. Iberoam. 16 (2) (2000), 243-279.

[23] N. Shanmugalingam, Harmonic functions on metric spaces, Illinois J. Math. 45 (2001), 10211050.

[24] N. Shanmuganlingam, D. Yang And W. Yuan, Newton-Besov Spaces and Newton-TriebelLizorkin spaces on metric measure spaces, Positivity 19 (2015), 177-220.

[25] D. YAnG, New characterizations of Hajtasz-Sobolev spaces on metric spaces, Sci. China Math. Ser. A 46 (5) (2003), 675-689.

[26] W. YUAN, Y. LU AND D. YANG, Fractional Hajtasz-Morrey-Sobolev spaces on quasi-metric measure spaces, Studia Math. 226 (2015), 95-122.

[27] W. YuAn, Y. Lu AND D. YAng, Several equivalent characterizations of fractional Hajtasz-MorreySobolev spaces, Appl. Math. J. Chinese Univ. 31 (2016), 343-354. 